\title{
Efeitos a curto prazo de "stents" não recobertos e recobertos com politetrafluoroetileno em aorta de suínos: um modelo experimental ${ }^{1}$
}

\author{
Marco Aurélio Grüdtner ${ }^{2}$, Adamastor Humberto Pereira ${ }^{3}$, Luiz Francisco Machado da Costa ${ }^{3}$, \\ Gilberto Gonçalves de Souza ${ }^{2}$, Rodrigo Argenta ${ }^{4}$, Joel Alex Longhi ${ }^{5}$
}

\begin{abstract}
Grüdtner MA, Pereira AH, Costa LFM, Souza GG, Argenta R, Longhi JA. Efeitos a curto prazo de "stents" não recobertos e recobertos com politetrafluoroetileno em aorta de suínos: um modelo experimental. Acta Cir Bras [serial online] 2004 Mar-Abr;19(2). Disponível em URL: http://www.scielo.br/acb.
\end{abstract}

RESUMO - Objetivo: Descrever um estudo experimental avaliando através da morfometria digital o espessamento intimal na parede arterial após o implante de "stents" metálicos auto-expansíveis recobertos ou não com politetrafluoroetileno (PTFE) na aorta de suínos. Métodos: Em três grupos de suínos jovens uma bainha introdutora de $12 \mathrm{~F}$ foi inserida na aorta abdominal distal. Os animais do grupo $\mathrm{I}(\mathrm{n}=5)$ foram considerados controle. Os animais do grupo II ( $n=10)$ receberam o implante de um stent metálico auto-expansível não recoberto. No grupo III (n=10) um stent auto-expansível recoberto com PTFE foi inserido. Após quatro semanas os animais foram sacrificados e os espécimes arteriais foram retirados, sendo o espessamento intimal quantificado pela análise morfométrica. Resultados: Na comparação entre os grupos I, II e III quanto às áreas da íntima, média e índice intimal, não foi observada variação estatisticamente significativa. Diferenças foram observadas entre os grupos em relação às áreas luminais proximais $(\mathrm{p}=0,0036)$ e distais $(\mathrm{p}=0,044)$. Através dos testes de comparação múltipla para Kruskal-Wallis foi identificada uma diferença entre os grupos I e II. Entretanto, quando essas variáveis foram controladas pelo fator peso (relação área luminal/peso), a diferença não foi mais observada. Conclusões: Nesse estudo a curto prazo, o revestimento de PTFE não esteve associado a adicional espessamento intimal além daquele promovido pelo dispositivo metálico em artérias de grande calibre e condições de alto fluxo.

DESCRITORES - Próteses vasculares. Politetrafluoroetileno. Aorta abdominal. Túnica íntima. Modelos animais.

\section{Introdução}

Estudos experimentais têm demonstrado que a parede arterial apresenta uma resposta multifatorial à lesão mecânica denominada de hiperplasia intimal. Topograficamente, essa resposta ocorre principalmente na túnica íntima e é caracterizada por proliferação celular e espessamento intimal que pode resultar em significativa redução da luz vascular ${ }^{1,2,3}$. A hiperplasia intimal é uma complicação relativamente freqüente após procedimentos endovasculares e pode ser considerada a principal causa de reestenose no seguimento de pacientes submetidos ao implante de "stents", uma vez que outro importante componente, o remodelamento geomé- trico, é minimizado pelo dispositivo intravascular $^{4,5,6,7,8}$.

Os "stents" recobertos com material protético estão sendo utilizados no tratamento da doença aterosclerótica oclusiva com o objetivo de limitar o espessamento intimal e, conseqüentemente a taxa de reestenose, melhorando a perviedade arterial a médio e longo prazo ${ }^{9,10,11}$. Entretanto, a presença do material protético pode estar associada a uma lesão adicional da parede arterial e a uma reação intimal superimposta àquela induzida pelo dispositivo intravascular ${ }^{12,}$ $13,14,15$

O propósito deste estudo foi avaliar, através da morfometria digital, o espessamento intimal na parede arterial imediata- mente proximal e distal ao implante de stents metálicos auto-expansíveis recobertos ou não com politetrafluoroetileno (PTFE) em um modelo experimental suíno.

\section{Métodos}

Esse experimento utilizou "stents" metálicos auto-expansíveis da série 316L, com configuração em "Z", de $30 \mathrm{~mm}$ de comprimento e $8 \mathrm{~mm}$ de diâmetro final, recobertos ou não com PTFE. O diâmetro escolhido foi baseado na avaliação prévia do calibre da aorta abdominal de suínos jovens que corresponde a aproximadamente $7 \mathrm{~mm}$ de diâmetro. Os stents foram confeccionados pelo Laboratório de Transformação Mecânica da Escola de

1. Trabalho realizado no Serviço de Cirurgia Vascular do Hospital de Clínicas de Porto Alegre, Universidade Federal do Rio Grande do Sul (UFRGS).

2. Médico Contratado do Hospital de Clínicas de Porto Alegre. Cirurgião vascular.

3. Professor Adjunto do Departamento de Cirurgia da UFRGS.

4. Médico Residente em Cirurgia Vascular do Hospital Nossa Senhora Conceição.

5. Médico Residente em Cirurgia Geral do Hospital de Clínicas de Porto Alegre. 
Engenharia da Universidade Federal do Rio Grande do Sul.

$\mathrm{O}$ projeto de pesquisa foi submetido ao Grupo de Pesquisa e Pós-Graduação do Hospital de Clínicas de Porto Alegre da Universidade Federal do Rio Grande do Sul para a apreciação dos aspectos éticos envolvidos no estudo.

Vinte e cinco suínos de raça mista (Landrace x Large White), com um peso médio de $20 \mathrm{~kg}$ foram utilizados neste estudo. Os procedimentos foram conduzidos sob anestesia geral inalatória através de um sistema aberto sem reinalação. Todos os animais foram medicados com midazolan intramuscular $(10 \mathrm{mg})$ e cefazolina $(1 \mathrm{~g})$ antes do procedimento. Uma solução endovenosa a 2,5\% de tiopental sódico (10 a $12 \mathrm{mg} / \mathrm{kg}$ ) foi utilizada como indutor anestésico e os animais foram ventilados com oxigênio e ar ambiente. A anestesia foi mantida com halotano e os animais receberam solução glico-fisiológica $(20 \mathrm{ml} / \mathrm{kg})$ para repor as perdas de fluidos. O experimento foi divido em duas fases:

\section{Fase 1}

A aorta abdominal distal e as artérias ilíacas foram minimamente dissecadas através de uma incisão retroperitoneal à esquerda.

No grupo I $(n=5)$, considerado o grupo controle, os animais foram submetidos a arteriotomia transversa da aorta abdominal distal e a manipulação arterial com uma bainha introdutora $12 \mathrm{~F}$.

No grupo II $(n=10)$, após uma arteriotomia transversa da aorta abdominal distal e a introdução de uma bainha introdutora 12 F, foi liberado um stent auto-expansível não recoberto na aorta abdominal infrarenal.

No grupo III ( $\mathrm{n}=10)$, após uma arteriotomia transversa da aorta abdominal distal e a introdução de uma bainha introdutora 12 F, foi liberado um stent auto-expansível recoberto com PTFE na aorta abdominal infra-renal.

Uma sutura contínua com polipropileno número 6-0 foi utilizada para fechamento das arteriotomias após a remoção da bainha introdutora $12 \mathrm{~F}$.

A adequada expansão do stent e a perviedade da aorta abdominal foi avaliada através de aortografia durante o procedimento.
Todos os animais receberam heparina sistêmica (100 UI/kg) cerca de 10 minutos antes da manipulação arterial. Nenhum agente anticoagulante ou anti-plaquetário foi utilizado após o procedimento.

Os animais foram alojados em duas baias com água corrente e alimentados com a mesma ração, balanceada para a idade e sem suplementação lipídica adicional por um período de 4 semanas.

\section{Fase 2}

Após 4 semanas os animais foram novamente anestesiados e submetidos a laparotomia mediana com exposição da aorta abdominal e das artérias ilíacas. Os espécimes cirúrgicos contendo a aorta abdominal infra-renal e as artérias ilíacas foram removidos em bloco após uma dose letal de tiopental sódico a 2,5\%. Para avaliação com a microscopia óptica, os espécimes cirúrgicos foram irrigados com solução de $\mathrm{NaCl}$ 0,9\% e fixados em solução de formalina tamponada neutra a $10 \%$. A seguir, os segmentos aorto-ilíacos foram seccionados transversalmente ao nível da aorta infra-renal e acima da trifurcação aórtica (grupo I) e junto às margens proximal e distal dos "stents" (grupos II e III). Os segmentos aórticos proximais e distais foram incluídos em blocos de parafina, seccionados em cortes de $4 \mu \mathrm{m}$ e corados com as técnicas de Hematoxilina-Eosina e Verhoeff (Figura 1).

As imagens dos cortes histológicos foram digitalizadas para a análise morfométrica através dos programas de morfometria e análise de imagem IMAGEPRO ${ }^{\circledR}$ PLUS - Versão 4.1 (Media Cybernetics - Silver Spring, USA) e IMAGE (Scion Corporation - USA). As imagens dos cortes histológicos foram digitalizadas a partir da microscopia óptica convencional: microscópio com lentes plano-acromáticas (ZEISS Axiostar - Alemanha) com fototubo ajustado para magnificação de 0,25 , câmera de circuito fechado colorida (câmera SONY DXC 151Japão) e placa de conversão analógica digital (IMAGE-PRO® PLUS CAPTURE KIT - Media Cybernetics - Silver Spring, USA), gerando arquivos de imagem $640 \mathrm{x}$ 480 pixels, 24 bits, em microcomputador. As imagens foram digitalizadas com aumento microscópico de 200 vezes e resolução final de 1 micrômetro por pixel, sendo a etapa de segmentação realizada em 200 aumentos. A planimetria das áreas do lúmen vascular, da túnica íntima e da túnica média foi realizada automatizadamente, sem a interferência do observador, mantido "cego" na mensuração.

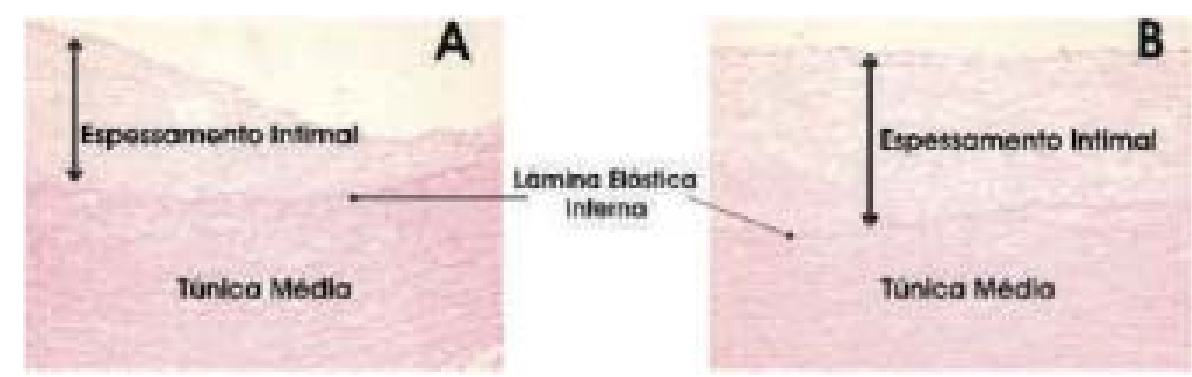

FIGURA 1 - Fotomicrografia de uma secção transversa da parede aórtica proximal (A) e distal (B) 4 semanas após o implante de um stent recoberto com PTFE. Observa-se espessamento intimal focal. A lâmina elástica interna (setas) separa a túnica íntima espessada da túnica média. Método da hematoxilina-eosina (200X).

A área luminal foi obtida por medida direta da área delimitada pelo endotélio; a da íntima, subtraindo-se a área luminal da delimitada pela lâmina elástica interna; a da túnica média, subtraindo-se a área luminal e da íntima da delimitada pela lâmina elástica externa.

Os resultados das medidas morfométricas das áreas da íntima e da média foram apresentados em números absolutos (milíme- tros quadrados), utilizando-se a média de oito medidas circunferenciais. O índice intimal (relação área intimal/área da túnica média $X$ 100) foi expresso em números relativos.

A análise estatística foi realizada pelo programa estatístico SPSS v.6.0 Statistical Package for Social Sciences for Windows (Microsoft - USA), através da estatística descritiva, utilizando-se a média e o desvio 
padrão para as variáveis paramétricas e a mediana e os intervalos inter-quartis (percentis 25-75) para as variáveis não paramétricas. Um valor de $\mathrm{p}<0,05$ foi considerado estatisticamente significativo.

\section{Resultados}

Na segunda fase do estudo foi identificada trombose da aorta abdominal infra-renal em quatro animais e estes foram excluídos da avaliação morfométrica. Um (01) caso de trombose ocorreu no grupo II e três (03) casos no grupo III. A avaliação macroscópica dos espécimes demonstrou que nas aortas pérvias os "stents" estavam firmemente aderidos à parede aórtica (Figura 2). Além disso, um fino revestimento translúcido foi observado, estendendo-se continuamente através das hastes nas extremidades dos "stents", na região central dos "stents" e sobre o PTFE nos "stents" revestidos. Na região central dos "stents" a cobertura era aparentemente descontínua tanto nos "stents" recobertos quanto nos não recobertos. Nas aortas trombosadas havia pouca integração das hastes dos "stents" à parede vascular. Em nenhum dos espécimes cirúrgicos pérvios observou-se macroscopicamente a presença de estreitamento luminal significativo na região central, nas hastes dos "stents" ou na transição entre o stent e a artéria.
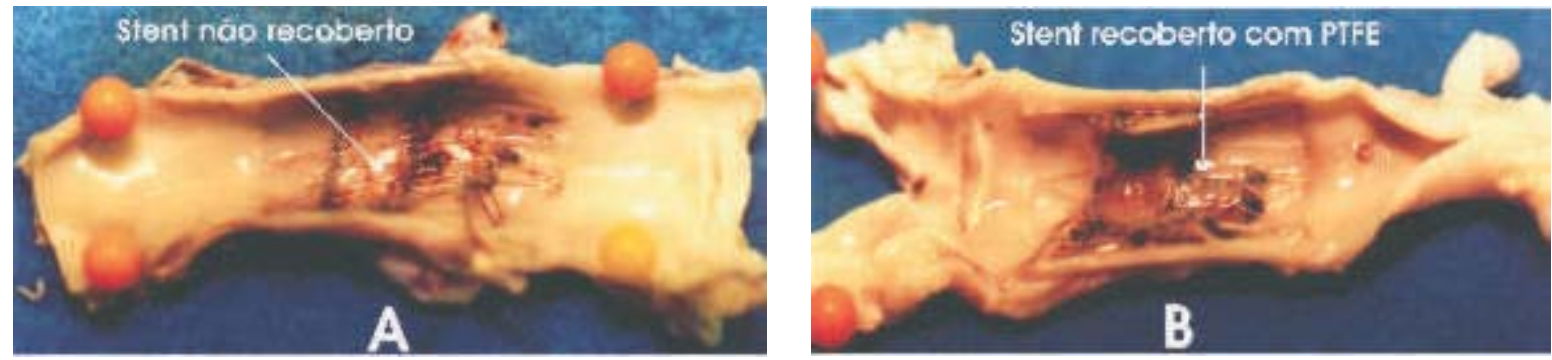

FIGURA 2 - Corte longitudinal de um espécime aórtico retirado 4 semanas após o implante de um stent não recoberto (A) e recoberto com PTFE (B).

TABELA 1 - Medidas morfométricas da parede aórtica

\begin{tabular}{ccccc}
\hline & Grupo I & Grupo II & Grupo III & p * \\
\cline { 2 - 5 } Área Proximal $\left(\mathbf{m m}^{2}\right)$ & 5,03 & 9,06 & 8,75 & $\mathbf{0 , 0 3 6} \dagger$ \\
Luminal & $(3,67-8,05)$ & $(8,04-14,34)$ & $(7,50-10,93)$ & \\
Intimal & 0,05 & 0,06 & 0,04 & 1,000 \\
Média & $(0,03-0,09)$ & $(0,02-0,13)$ & $(0,03-0,19)$ & \\
Índice Intimal & 4,90 & 4,88 & 4,02 & 0,151 \\
& $(4,64-5,12)$ & $(4,27-5,57)$ & $(3,77-4,86)$ & \\
Área Distal (mm $\left.{ }^{2}\right)$ & 0,1089 & 0,00982 & 0,01073 & 0,851 \\
Luminal & $(0,0726-0,01755)$ & $(0,00486-0,02647)$ & $(0,00744-0,03876)$ & \\
Intimal & 8,71 & & & \\
& $(7,99-8,98)$ & $(10,43-17,90)$ & $(9,38-11,41)$ & \\
Média & 0,01 & 0,10 & 0,09 & 0,195 \\
& $(0,001-0,11)$ & $(0,04-0,14)$ & $(0,02-0,11)$ & \\
Índice Intimal & 4,78 & 5,35 & 5,64 & 0,068 \\
& $(4,34-4,86)$ & $(5,00-5,80)$ & $(4,46-6,63)$ & \\
& $(0,00180$ & 0,01502 & 0,01763 & 0,282 \\
& & 12,38 & $(0,00404-0,02207)$ & \\
\hline
\end{tabular}

Medianas com intervalos inter-quartis (25-75) entre parênteses.

* Valores de p encontrados na comparação entre os grupos (Teste de Kruskal-Wallis). $\uparrow$ Significante estatisticamente.

A análise das medidas morfométricas da parede arterial foi realizada dentro de cada grupo, entre as regiões da parede arterial proximal e distal pelo teste T de Wilcoxon, e entre os grupos I, II e III pelo teste de Kruskal-Wallis.
A Tabela 1 apresenta os resultados das medidas morfométricas de secções transversais da parede aórtica proximal e distal de cada grupo.

Quando os espécimes da parede arterial proximal e distal foram comparados, ne- nhuma diferença estatisticamente significativa foi encontrada entre as áreas luminal, intimal e média ou índice intimal dentro de cada grupo. Na comparação entre os grupos I, II e III quanto às áreas intimal, média e índice intimal, também não se observou variação estatisticamente significativa. Foram identificadas diferenças entre os grupos quanto às áreas luminais proximais $(\mathrm{p}=0,036)$ e distais $(\mathrm{p}=0,044)$. Pelo teste de comparações múltiplas para Kruskal-Wallis (Teste de Dunn) identificouse a diferença nos grupos I e II. Entretanto, quando essas variáveis foram controladas pelo fator peso (relação área luminal/peso) a diferença não foi mais observada.

\section{Discussão}

O tratamento da doença aterosclerótica oclusiva por métodos intravasculares representa uma alternativa menos invasiva que a cirurgia convencional. Entretanto, o desenvolvimento de um estreitamento luminal nas áreas submetidas a angioplastia transluminal percutânea e/ou implante de "stents" tem sido o principal problema, limitando a perviedade a médio e longo prazo desses procedimentos.

Palmaz observou que no processo de incorporação de "stents" vasculares a camada inicial de trombo que reveste o stent é substituída progressivamente por tecido 
fibromuscular, inicialmente em torno das extremidades do stent e, em seguida, expandindo-se excentricamente ${ }^{16}$.

Hoffman e col em um estudo clínico com ultrasonografia intravascular demonstraram que os "stents" estão relacionados a alterações bem definidas na parede arterial. Em artérias coronárias submetidas ao implante de "stents" de Palmaz-Schatz ocorreram alterações a médio prazo nas margens entre o stent e a artéria que posteriormente estenderam-se para os segmentos adjacentes nas margens proximal e distal entre o stent e a artéria por no mínimo 5 a $10 \mathrm{~mm}$. Além disso, a proliferação e o remodelamento observados na parede arterial adjacente ao stent foram similares àqueles encontrados na região central do corpo do stent ${ }^{17}$.

Embora a maioria das publicações disponíveis sobre procedimentos intravasculares inclua estudos sobre artérias coronárias, os mecanismos de reestenose em outras artérias humanas são considerados similares ${ }^{18}$. Por outro lado, a análise morfométrica após o implante de "stents" na aorta abdominal é pouco mencionada ${ }^{9,15,19}$.

Em nosso estudo, não pudemos demonstrar pela análise morfométrica diferenças estatisticamente significativas entre os grupos, em relação ao espessamento intimal, entre os segmentos arteriais proximais e distais aos "stents". A diferença observada entre as áreas luminais proximais e distais do grupo I e II pode ser explicada pelo maior ganho de peso dos animais do grupo II. De qualquer maneira, parece que a curto prazo a reação intimal secundária ao implante de "stents" em vasos de grande calibre e em situações de alto fluxo não se estende além das margens com a artéria nativa. Em um recente estudo com um modelo suíno aterosclerótico, Castaneda e col não encontraram diferenças na espessura da íntima e média nas regiões proximais e distais da aorta e de artérias ilíacas submetidas ao implante de "stents" de nitinol recobertos com poliéster ${ }^{15}$.

Por outro lado, Yuan e col observaram maior hiperplasia intimal e estreitamento luminal após o implante de "stents" metálicos em artérias ilíacas de suínos do que após a angioplastia isolada. Naquele estudo, o revestimento de PTFE não evitou o desenvolvimento de hiperplasia intimal na artéria proximal e distal junto ao stent após 4 semanas $^{13}$. Já em outro estu- do, White e col observaram mínimo espessamento intimal, limitado as regiões dos ganchos proximais, e nenhuma evidência de estenose ou oclusão da aorta em ovelhas após o implante de "stents" recobertos com poliéster ${ }^{20}$.

Em nosso estudo, a avaliação macroscópica das aortas pérvias demonstrou que os "stents" estavam firmemente aderidos à parede arterial. Além disso, um fino revestimento translúcido foi observado ao longo das extremidades dos "stents" e na região central e sobre o PTFE nos "stents" recobertos. Na área central dos "stents" o revestimento translúcido era aparentemente descontínuo. Nas aortas trombosadas houve pouca integração dos "stents" à parede vascular, sugerindo uma expansão incompleta ou uma retração do dispositivo. Existem evidentes limitações na extrapolação de estudos animais para humanos que devem ser considerados. Apesar das semelhanças anátomo-fisiológicas, a aorta suína pode responder de maneira diferente da aorta humana. Além disso, as modificações causadas pela presença do stent podem ser diferentes em uma aorta aterosclerótica. Infelizmente, não existem modelos experimentais adequados de aterosclerose aórtica em grandes animais. Embora não tenham ocorrido lesões reestenóticas no período de estudo, em concordância com outros estudos de curto seguimento, parece mais adequado o desenvolvimento de um modelo experimental com um tempo maior de acompanhamento dos animais. Entre os estudos clínicos deve-se mencionar o de Marin e col que analisaram "stents" recobertos com PTFE retirados do segmento aorto-ilíaco-femoral no momento da conversão cirúrgica por falência do stent ou no exame anátomo-patológico pósmorte de sete pacientes. Os "stents" examinados seis semanas após o implante demonstraram estar firmemente aderidos à parede arterial e apresentavam uma neoíntima bem desenvolvida na interface entre o stent e a artéria. Em uma reconstrução em falência uma estenose foi demonstrada na margem distal do stent, secundária a significativo espessamento intimal ${ }^{21}$. Em outro estudo, Marin e col. avaliaram a relação entre o revestimento de PTFE e o desenvolvimento de hiperplasia intimal em 12 "stents" implantados em artérias ilíacas comuns de pacientes de alto risco cirúrgi- co. O diâmetro luminal médio no segmento recoberto foi significativamente maior do que no segmento não recoberto em angiografias realizadas 4 a 6 meses após o procedimento, sugerindo que o revestimento pudesse evitar a reestenose ${ }^{22}$.

\section{Conclusão}

$\mathrm{Na}$ incorporação de um stent vascular, o desenvolvimento de uma neo-íntima estável e o revestimento por um endotélio funcionante são consideradas as características mais importantes para a completa incorporação e manutenção da perviedade a longo prazo do dispositivo. Os resultados observados por nós sugerem que, pelo menos a curto prazo, o revestimento com PTFE não está associado a um adicional espessamento intimal além daquele promovido pelo dispositivo intravascular, em artérias de grande calibre e em condições de alto fluxo. Esses resultados podem ter implicações no sentido de melhorias tecnológicas nos "stents", já que o principal objetivo é a manutenção da perviedade vascular associada a melhor incorporação possível na parede arterial com mínima reação intimal.

\section{Referências}

1. Castagna MT, Mintz GS, Leiboff BO, Ahmed JM, Mehran R, Satler LF, Kent KM, Pichard AD, Weissman NJ.The contribution of mechanical problems to in-stent restenosis: an intravascular ultrasonographic analysis of 1090 consecutive in-stent restenosis lesions. Am Heart J 2001;142(6):970-4.

2. Sullivan TM, Ainsworth SD, Langan EM, Taylor S, Snyder B, Cull D, Youkey J, Laberge M. Effect of endovascular stent strut geometry on vascular injury, myointimal hyperplasia, and restenosis. J Vasc Surg 2002;36(1):143-9.

3. Schwartz RS. Pathophysiology of restenosis: interaction of thrombosis, hyperplasia, and/or remodeling. Am J Cardiol 1998;81(7A):14e-7e.

4. Inoue S, Koyama H, Miyata T, Shigematsu H. Pathogenetic heterogeneity of in-stent lesion formation in human peripheral arterial disease. J Vasc Surg 2002;35(4):672-8.

5. Yoshitomi Y, Kojima S, Yano M, Sugi T, Matsumoto Y, Saotome M, Tanaka K, Endo M, Kuramochi M. Does stent design affect probability of restenosis? A randomized trial-comparing Multilink stents with GFX stents. Am Heart J 2001;142(3):445-51.

6. Gomez-Hospital JA, Cequier A, FernandezNofrerias E, Mauri J, Garcia del Blanco B, Iraculis E, Jará F, Esplugas E. The treatment of intra-stent restenosis. The current 
situation and future outlook. Rev Esp Cardiol 1999;52(12):1130-8.

7. Mintz GS, Mehran R, Waksman R, Pichard AD, Kent KM, Satler LF, Leon MB. Treatment of in-stent restenosis. Semin Interv Cardiol 1998;3(2):117-21.

8. Post MJ, De Smet BJGL, Van Der Helm Y, Borst C, Kuntz RE. Arterial remodeling after balloon angioplasty or stenting in an atherosclerotic experimental model. Circulation 1997;96:996-1003.

9. Weatherford DA, Ombrellaro MP, Schaeffer DO, Stevens SL, Sackman JE, Freeman MB, Goldman MH. Healing characteristics of intraarterial stent grafts in an injured artery model. Ann Vasc Surg 1997;11(1):54-61.

10. Ohki T, Marin, ML, Veith FJ, Ohki M, Soundararajan K, Sanchez LA, Parsons RE, Lyon RT, Yamazaki Y. Anastomotic intimal hyperplasia: a comparison between conventional and endovascular stent graft techniques. J Surg Res 1997;69(2):255-67.

11. Ombrellaro MP, Stevens SL, Kerstetter K, Freeman MB, Goldman MH. Healing characteristics of intraarterial stented grafts: Effect of intraluminal position on prosthetic graft healing. Surgery 1996;120:60-70
12. Sick PB, Brosteanu O, Niebauer J, Hehrlein C, Schuler G. Neointima formation after stent implantation in an experimental model of restenosis: polytetrafluoroethylenecovered versus uncovered stainless steel stents. Heart Dis 2002; 4(1):18-25.

13. Yuan JG, Ohki T, Marin ML, Quintos RT, Krohn DL, Beitler JJ, Veith FJ. The effect of nonporous PTFE-covered stents on intimal hyperplasia following balloon arterial injury in minipigs. J Endovasc Surg 1998;5(4):349-58.

14. Schurmann K, Vorwerk D, Uppenkamp R, Klosterhalfen B, Bucker A, Gunther RW. Iliac arteries: plain and heparin-coated dacron-covered stent-grafts compared with noncovered metal stents- an experimental study. Radiology 1997;203:55-63.

15. Castaneda F, Ball-Kell SM, Wyffels PL, Young K, Li R. Assessment of a polyestercovered nitinol stent in an atherosclerotic swine model. J Vasc Interv Radiol 2000;11:483-91.

16. Palmaz JC. Intravascular stents: tissue-stent interactions and design considerations. Am J Roentgenol 1993;160:613-8.

17. Hoffmann R, Mintz GS, Dussaillant GR, Popma JJ, Pichard AD, Satler LF, Kent KM, Griffin J, Leon MB. Patterns and mechanisms of in-stent restenosis: a serial intravascular ultrasound study. Circulation 1996;94:1247-54.

18. Byer A, Ussia G, Galleti G. Autologous vein lined and covered stents in swine arteries. J Cardiovasc Surg 1998;39:393-8.

19. Lee KW, Park JH, Chung JW, Kim WS, Lee W, Yeon KM. Short-term effects of a new intravascular nitinol stent in canine arteries. Invest Radiol 1999;34(5):367-73.

20. White JG, Mulligan NJ, Gorin DR, D'agostino R, Yucel K, Menzoian JO. Response of normal aorta to endovascular grafting. Arch Surg 1998;133:246-9.

21. Marin ML, Veith FJ, Cynamon J, Sanchez LA, Bakal CW, Suggs WD, Lyon RT, Schawartz ML, Parsons RE, Wengerter KR, et al. Human transluminally placed endovascular stented grafts: preliminary histopathologic analysis of healing grafts in aortoiliac and femoral artery occlusive disease. J Vasc Surg 1995;21(4):595-603.

22. Marin ML, Veith FJ, Cynamon J, Parsons RE, Lyon RT, Suggs WD, Bakal CW, Waahl S, Sanchez LA, Yuan JG, Ohki T. Effect of polytetrafluoroethylene covering of Palmaz stents on the development of intimal hyperplasia in human iliac arteries. J Vasc Interv Radiol 1996;7(5):651-6.

Grüdtner MA, Pereira AH, Costa LFM, Souza GG, Argenta R, Longhi JA. Short-term effects of polytetrafluoroethylene covered and uncovered metallic "stents" in pig aorta: an experimental model. Acta Cir Bras [serial online] 2004 MarApr;19(2). Available from URL: http://www.scielo.br/acb.

ABSTRACT - Purpose: To report an experimental study evaluating, through digital morphometry, the intimal thickening of the arterial wall after the implant of auto-expandable stainless steel stents covered or not with polytetrafluoroethylene (PTFE) in the pig aorta. Methods: In three groups of pigs a $12 \mathrm{~F}$ sheath was inserted in distal abdominal aorta. Group I animals $(n=5)$ served as control. Group II animals $(n=10)$ received an auto-expandable uncovered metallic stent. On group III animals $(n=10)$ an auto-expandable stent covered with PTFE was inserted. After four weeks the animals were killed, the specimens were harvested and the intimal thickening was quantified by morphometric analysis. Results: In the comparison among groups I, II and III referring to intimal, medial area and intimal index, it was not observed statistically significant variation. Differences were identified among groups referring to luminal proximal $(p=0,036)$ and distal areas $(\mathrm{p}=0,044)$. Through multiple comparison tests for Kruskal-Wallis it was identified a difference between groups I and II. However, when these variables were controlled by weigh factor (luminal area/weigh relation), this difference was not observed anymore. Conclusion: In this short term study, the PTFE covering is not associated to further intimal thickening besides that promoted by the metallic mesh in large arteries and in high flow conditions.

KEY WORDS - Blood vessel prothesis. Polytetrafluoroethylene. Aorta, abdominal. Tunica intima. Models, animal.

Conflito de interesse: nenhum

Fonte de financiamento: nenhuma

Correspondência:

Dr. Marco Aurélio Grüdtner

Rua Ramiro Barcelos 2350/sala 600

90035-003 Porto Alegre - RS

Tel.: (51)3316-8232

mgrudtner@terra.com.br

Data do recebimento: $12 / 01 / 2004$

Data da revisão: 25/01/2004

Data da aprovação: 08/02/2004

*Fotos coloridas disponíveis em www.scielo.br/acb 